\title{
Selective intermanual transfer of adaptive effects during adaptation to prism'
}

SAMUEL C. MCLAUGHLIN AND JOHN L. BOWER

TUFTS UNIVERSITY

\begin{abstract}
Abstraet
Complete adaptation to prism has the appearance of a unitary phenomenon; but, at intermediate stages of adaptation, the total adaptive effect can be analyzed into two components which combine additively. One of these two components transfers $100 \%$ from adapted hand to unadapted hand, whereas the other does not transfer at all. It is concluded that one is a change in the apparent position of the visual stimulus while the other is a change in the felt position of the adapted hand.

\section{Introduction}

McLaughlin \& Rifkin (1965) showed that the sensorymotor change accompanying adaptation to prism can be analyzed into two components which combine additively in varying proportions during the course of adaptation. One of these components-a parallel shift in visual localization and judgments of "straight ahead"-was interpreted as a shift in the entire egocentric frame of reference. Harris (1965) suggested that this phenomenon might better be interpreted as a change in the felt position of the hand. In order to choose between these two interpretations, it is necessary to know the extent to which this component of the adaptive process transfers from adapted hand to unadapted hand. The present experiment was designed to provide that information.

\section{Apparatus}

$\mathrm{S}$ sat with his forearms resting symmetrically on a table. He viewed the visual target, a vertical luminous white line $3 \mathrm{~cm}$ high and $0.2 \mathrm{~cm}$ wide, from a distance of $33 \mathrm{~cm}$, his head being held immobile by means of a bite board containing his dental impression. His task was to move a pointer along a horizontal track perpendicular to his line of sight. The track was directly beneath the visual display. The pointer was tapered upward, its tip being $0.2 \mathrm{~cm}$ lower than the bottom edge of the white line.

\section{Procedure}

With the room darkened and the visual stimulus turned off, wedge prisms of 20 prism diopters were placed in front of S's eyes. S was instructed to set the pointer straight ahead, first with one hand and then with the other hand. The visual stimulus was then turned on, and two settings "on the line" were made, one with each hand. Finally, the visual display was illuminated, enabling $S$ to see his hand and the pointer as well as the line. He was instructed to set the pointer on the line using his right hand. The hand not used in making a setting was out of S's field of view。

This sequence of five settings was repeated four times, except that the final setting with the display illuminated

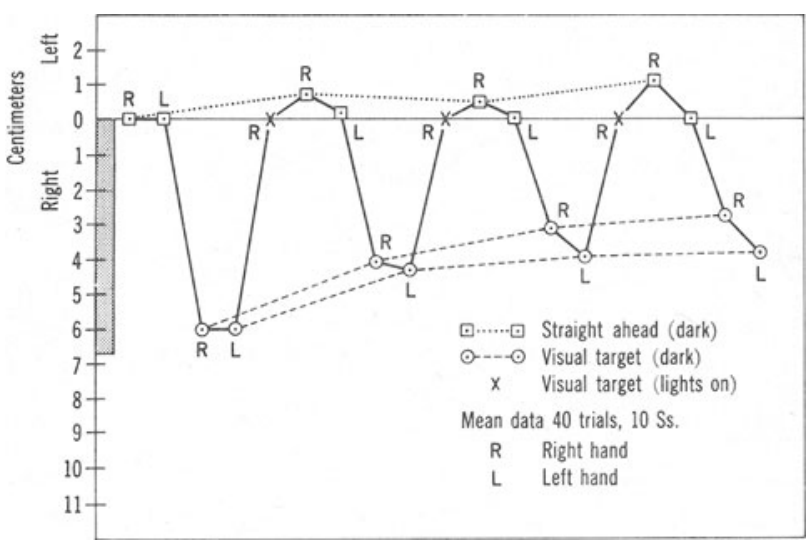

Fig. 1. Mean data for $10 \mathrm{Ss}$. Each point represents the mean of 40 settings: four by each $S$. The zero point on the ordinate represents the veridical position of the visual target. Settings above zero represent settings to $S$ 's left; settings below zero represent settings to S's right. The thickened portion of the ordinate represents the direction and magnitude of the prism deviation. In half the trials, the left hand settings were made first; and in half the trials the prism deviation was to the left rather than to the right; but the data have been transposed in order to make it possible to combine the results of all trials.

was omitted, so that 19 settings were made in a single trial. The visual target, which had been left off during the first two settings, was kept on during the remainder of the trial.

In half of the trials, "straight ahead" and "line" settings (in the dark) were made with the left hand first, and in half of the trials they were made with the right hand first; but the right hand was used during all "adaptation" settings (i.e., with the display illuminated).

Each $S$ went through four trials in a single session: two with prismatic deviation to the right (as in Fig. 1) and two with prismatic displacement to the left; twice with right hand first, and twice with left hand first. The combinations of prism orientation and right or left hand first were varied systemically so that each combination appeared an equal number of times. To provide approximate compensation for sequential effects during an experimental session, the two prism orientations were presented in sequences of RLLR and LRRL with alternate Ss.

\section{Results}

The first step in processing the data was to carry out two transformations: the results from trials in which left-hand settings had been made first were tabulated with right-hand settings first; and the results from trials with prism deviation to the left were tabulated with the direction of each setting from zero 
reversed. (This reversal was not applied to the first two settings of each trial, since those two were unaffected by the prism deviation.) These transformations made it possible to combine the data for all trials, and this has been done in Fig. 1. In the following analysis, all numbers refer to the mean data shown in Fig. 1.

Shift in straight ahead. For the right hand, judgments of straight ahead shifted $0.9 \mathrm{~cm}$ in a direction opposite to the prism deviation. For the left hand, judgments of striaight ahead shifted $0.1 \mathrm{~cm}$ in the same direction. The shift for the right hand was significantly different from zero $(\mathrm{N}=40 ; \mathrm{C} . \mathrm{R}=3.67 ; \mathrm{p}<.001)$; that for the left hand was not significantly different from zero (C.R=0.29)。

Reduction in disparity. For the right hand, the prism-induced disparity between "line" and "straight ahead" settings diminished by $2.4 \mathrm{~cm}$ during the course of adaptation. For the left hand, the disparity also diminished by $2.4 \mathrm{~cm}$. Both changes are different from zero at $\mathrm{p}<.001$.

Thus, the reduction in disparity transferred exactly $100 \%$ from adapted hand to unadapted hand; and, with an error of only $1 \mathrm{~mm}$, the shift in straight ahead (and hence the parallel shift in "straight ahead" judgments and visual localization) did not transfer at all.

\section{Discussion}

These results confirm Harris' interpretation of the McLaughlin and Rifkin data in showing that the parallel shift in visual localization and "straight ahead" judgments is a change in felt position of the hand rather than a shift in the egocentric frame of reference. However, there are at least three lines of evidence which suggest that it is inadvisable to rule out the possibility of a shift in egocentric frame of reference during adaptation to prism. First, Ss make the anomalous "straight ahead" settings even with hand and pointer seen. Second, preliminary evidence suggests that the change in straight ahead transfers intermanually during the later stages of the adaptive process. Third, Ss in the McLaughlin and Rifkin type of experiment sometimes report a feeling of a change in "orientation in space" coincident with an observed shift in judgments of "straight ahead."

The other adaptive mechanism-the reduction in disparity between "line" and "straight ahead" settings-is a process in which the visual target approaches subjective straight ahead. This fact, coupled with the finding that it transfers fully from adapted hand to unadapted hand, suggests that it is (in terms of S's visual experience) a change in the apparent position of the visual target. This is evidently the adaptive effect which von Helmholtz observed: a "change in the judgment of the direction of the gaze" (Southall translation, 1924, p. 246).

\section{References}

von Helmholtz, H. Handbuch der Pysiologischen Optik (3rd ed.) Hamburg: Voss, 1910. Transl. by J. P. C. Southall: The Optical Society of America, 1924.

McLaughlin, S. C., \& Rifkin, K. I. Change in straight ahead during adaptation to prism. Psychon. Sci., 1965, 2, 107-108.

McLaughlin, S. C., \& Bower, J. L. Auditory localization and judgments of straight ahead during adaptation to prism. Psychon. Sci., 1965, 2, 283-284.

Harris, C. S. Comment on "Change in straight ahead during adaptation to prism." Psychon. Sci., 1965, 2, 285-286.

\section{Note}

1. This research was carried out under Contract MD-2714 with the Behavioral Sciences Research Branch of the U. S. Army Medical Research and Development Command. 